International Journal of Physical Research, $7(2)(2019) 106-110$
International Journal of Physical Research
SPC
Website: www.sciencepubco.com/index.php/IJPR
Research paper

\title{
Soliton solutions of coupled higgs field equation via the trial equation method
}

\author{
S. Subhaschandra Singh * \\ Physics Department, Imphal College, Imphal - 795001,Manipur, India \\ *Corresponding authorE-mail: subhasic@yahoo.co.in
}

\begin{abstract}
In this present work, the Coupled Higgs Field Equation, which is a nonlinear evolution equation (NLEE), is reduced to a nonlinear ordinary differential equation (NLODE) using transformations of variables and functions and the Trial Equation Method is applied for establishing Soliton Solutions of the coupled equation.
\end{abstract}

Keywords: Gauge Boson; Scalar M eson; Soliton; Trial Equation Method.

\section{Introduction}

Since a few recent decades, investigation of nonlinear evolution equations (NLEEs) is becoming an important area of research as they have a variety of applications in various branches of social and scientific disciplines like Ecology, Social Dynamics, Financial Mathematics, Engineering and many branches of Physics such as Biophysics, Chemical Physics, Fibre Optics, Fluid Mechanics, Neuro-physics, Particle Physics, Solid State Physics and many more. Many powerful and efficient methods of finding exact solutions of NLEEs have been proposed so far and the Trial Equation Method [ 1 - 5] is one of them. Many authors have successfully used the method in finding exact solutions of a number of NLEEs. In the present paper, soliton solutions of the Coupled Higgs Field Equation [ 6 - 10 ] are being obtained using the Trial Equation Method.

The Coupled Higgs Field Equation describes system of conserved scalar nucleons interacting with neutral scalar mesons in particle physics. This coupled equation has applications in the studies of Field Theory and Electromagnetic waves as well. This coupled equation introduces the Higgs field to illustrate the mechanism of generation of mass for Gauge Bosons. The Coupled Higgs Field Equation is generally expressed as the following pair of NLEEs

$u_{t t}-u_{x x}-\beta u+\gamma|u|^{2} u-2 u v=0$

and

$\mathrm{v}_{\mathrm{tt}}+\mathrm{v}_{\mathrm{xx}}-\gamma\left(|\mathrm{u}|^{2}\right)_{\mathrm{xx}}=0$

Here, $x$ and $t$ are spatial and temporal variables respectively, the function $u(x, t)$ is a complex scalar nucleon field, the function $v(x, t)$ is a real scalar meson field, $\beta$ and $\gamma$ are arbitrary real constants and the subscripts denote partial differentiations with respect to them. Using the Trial Equation Method, the above coupled NLEE is to be solved to obtain some soliton solutions.

\section{Outlines of trial equation method}

In this section, the outlines of the Trial Equation Method are briefly presented. The main steps are

Step 1:

In a given nonlinear partial differential equation of a function $u$ of two real variables $x$ (spatial) and $t$ (temporal) in the form

$P\left(x, t, u, u_{x}, u_{t}, u_{x x}, u_{t t}, u_{x t} \ldots\right)=0$

where $\mathrm{P}$ is a polynomial of the shown variables and the subscripts denote partial differentiations with respect to them, the equation is first transformed into a nonlinear ordinary differential equation in the form

$\mathrm{Q}\left(\xi, \mathrm{U}, \mathrm{U}^{\prime}, \mathrm{U}^{\prime \prime}, \mathrm{U}^{\prime \prime \prime}, \ldots\right)=0$ 
where $\mathrm{Q}$ is also a polynomial of the newly introduced variable $\xi=\mathrm{x}-\mathrm{ct}$, the newly introduced function $\mathrm{U}(\xi)$ and various derivatives of $\mathrm{U}(\xi)$. Here, c is a real constant (generally the constant speed of the associated wave),

$\mathrm{U}^{\prime}=\frac{\mathrm{dU}}{\mathrm{d} \xi}, \mathrm{U}^{\prime \prime}=\frac{\mathrm{d}^{2} \mathrm{U}}{\mathrm{d} \xi^{2}}$ etc.

If all the terms of Eq.(4) contain derivatives, the equation is to be integrated once or more until one term does not contain derivative(s) whilst the integration constant(s) are to be chosen as zero.

Step 2:

We assume a Trial Equation in the form

$\left(U^{\prime}\right)^{2}=F(U)=\sum_{j=0}^{m} a_{j} U^{j}$

where $a_{j}(j=0,1,2,3, \ldots m)$ are constants to be determined.

Substitution of Eq. (5) and other derivatives of $U$ such as $U^{\prime}, U^{\prime \prime}$ etc. into Eq. (4) will yield a polynomial G(U) of $U$. Then, the value of $m$ can be determined from the balancing principle. Next, setting the various coefficients in the polynomial G(U) to zero separately, we will obtain a system of algebraic equations. Solving such system of equations, we can determine the values of $a_{j}(j=0,1,2,3, \ldots \mathrm{m})$ and the constant c.

Step 3:

We can write Eq. (5) in integral form as

$\pm\left(\xi-\xi_{0}\right)=\int \frac{\mathrm{dU}}{\sqrt{\mathrm{F}(\mathrm{U})}}$

where $\xi_{0}$ is a constant?

Classifying the roots of the function $\mathrm{F}(\mathrm{U})$ and evaluating the integral in Eq. (6), we can obtain exact solutions of Eq.(3).

\section{Application of trial equation method to coupled higgs field equation}

In this section, the Trial Equation Method is being applied to seeking exact solutions of Coupled Higgs Field Equation, thereby yielding some soliton solutions and some periodic solutions.

Let us first reduce the Coupled Higgs Field Equation to two simultaneous nonlinear ordinary differential equations. To do this, let us introduce new functions with new variables as in the following.

$u(x, t)=U(\xi) e^{-(k x-\omega t)}$

and

$\mathrm{v}(\mathrm{x}, \mathrm{t})=\mathrm{V}(\xi)$

where $\mathrm{U}(\xi)$ and $\mathrm{V}(\xi)$ are real functions of a new variable $\xi$ defined by $\xi=\mathrm{x}-\mathrm{ct}$ and $\mathrm{c}, \mathrm{k}, \omega$ are constants to be determined.

Using the newly introduced variable and the functions, we write Eq. (1) as

$\left(c^{2}-1\right) U^{\prime \prime}+2 i(k-\omega c) U^{\prime}+\left(k^{2}-\omega^{2}-\beta\right) U+\gamma U^{3}-2 U V=0$

where

$\mathrm{U}^{\prime}=\frac{\mathrm{dU}}{\mathrm{d} \xi}, \mathrm{U}^{\prime \prime}=\frac{\mathrm{d}^{2} \mathrm{U}}{\mathrm{d} \xi^{2}}$, etc.

Imaginary part of Eq.(9) yields $\mathrm{k}=\omega \mathrm{c}$ and, hence, we re-write Eq.(9) as

$\left(c^{2}-1\right) U^{\prime \prime}+\left(k^{2}-\omega^{2}-\beta\right) U+\gamma U^{3}-2 U V=0$.

Further, Eq. (2) can also be written as

$\left(c^{2}+1\right) \frac{d^{2} V}{d \xi^{2}}=\gamma \frac{d^{2}\left(U^{2}\right)}{d \xi^{2}}$.

Integrating twice with respect to $\xi$ and choosing the integration constants as zero, we write

$\mathrm{V}=\frac{\gamma \mathrm{U}^{2}}{\mathrm{c}^{2}+1}$.

Substituting Eq.(11) into Eq.(10) and using k = $\omega c$, we write

$\left(c^{4}-1\right) U^{\prime \prime}+\left\{\left(c^{4}-1\right) \omega^{2}-\beta\left(c^{2}+1\right)\right\} U+\gamma\left(c^{2}-1\right) U^{3}=0$.

Now, $U(\xi)$ can be solved from Eq.(12) and then substituting in Eq. (11), we can obtain $V(\xi)$ also. Then, substituting $U(\xi)$ and $V(\xi)$ into Eqs. (7) And (8) and using $\xi=x-c t$, we can obtain the solutions $u(x, t)$ and $v(x, t)$ of Eqs. (1) and (2) respectively.

Now, recalling Eq. (5) and using derivatives of $U(\xi)$, we balance $U^{\prime \prime}$ with $U^{3}$ to yield 
$\mathrm{m}=4$. Thus, we re-write Eq. (5) as

$\left(\mathrm{U}^{\prime}\right)^{2}=\mathrm{F}(\mathrm{U})=\mathrm{a}_{0}+\mathrm{a}_{1} \mathrm{U}+\mathrm{a}_{2} \mathrm{U}^{2}+\mathrm{a}_{3} \mathrm{U}^{3}+\mathrm{a}_{4} \mathrm{U}^{4}$

Differentiating both sides of Eq. (13) with respect to $\xi$ and then dividing the results by $2 U^{\prime}$, we obtain

$U^{\prime \prime}=\frac{1}{2} F^{\prime}(U)=\frac{1}{2} a_{1}+a_{2} U+\frac{3}{2} a_{3} U^{2}+2 a_{4} U^{3}$.

Substituting Eq. (14) into Eq. (12), we obtain

$\left(c^{4}-1\right)\left(\frac{a_{1}}{2}+a_{2} U+\frac{3}{2} a_{3} U^{2}+2 a_{4} U^{3}\right)+\left\{\left(c^{4}-1\right) \omega^{2}-\left(c^{2}+1\right) \beta\right\} U$

$+\gamma\left(c^{2}-1\right) U^{3}=0$

Equating the various coefficients of different powers of $U$ to zero, we obtain the following system of algebraic equations.

$\frac{1}{2}\left(c^{4}-1\right) a_{1}=0$,

$\left(c^{4}-1\right) a_{2}+\left(c^{4}-1\right) \omega^{2}-\left(c^{2}+1\right) \beta=0$,

$\frac{3}{2}\left(c^{4}-1\right) a_{3}=0$,

and

$2\left(c^{4}-1\right) a_{4}+\gamma\left(c^{2}-1\right)=0$.

Solving the above system of equations, we obtain

$a_{1}=0, a_{2}=\frac{\beta}{c^{2}-1}-\omega^{2}$,

$a_{3}=0$ and $a_{4}=-\frac{\gamma}{2\left(c^{2}+1\right)}$.

Substituting the values of these coefficients into Eqns.(6) and (13), we obtain

$\pm\left(\xi-\xi_{0}\right)=\int \frac{d U}{\sqrt{a_{0}+\frac{\beta-\left(c^{2}-1\right) \omega^{2}}{c^{2}-1} U^{2}-\frac{\gamma}{2\left(c^{2}+1\right)} U^{4}}}$

If we set $a_{0}=0$ in Eq.(15), then integrating with respect to $U$, we can obtain $U(\xi)$.

Substituting the expression for $U(\xi)$ thus obtained into Eq.(7), we obtain a bright and a singular soliton solutions of Eq.(1) as

$u(x, t)= \pm \sqrt{\frac{2\left\{\beta\left(c^{2}+1\right)-\left(c^{4}-1\right) \omega^{2}\right\}}{\gamma\left(c^{2}-1\right)}} \times \operatorname{sech}\left[\sqrt{\frac{\beta-\left(c^{2}-1\right) \omega^{2}}{c^{2}-1}}\left(x-c t-x_{0}\right)\right] \times e^{-i(k x-\omega t)}$

and

$u(x, t)$

$= \pm \sqrt{-\frac{2\left\{\beta\left(c^{2}+1\right)-\left(c^{4}-1\right) \omega^{2}\right\}}{\gamma\left(c^{2}-1\right)}} \times \operatorname{cosech}\left[\sqrt{\frac{\beta-\left(c^{2}-1\right) \omega^{2}}{c^{2}-1}}\left(x-c t-x_{0}\right)\right] \times e^{-i(k x-\omega t)}$

where $x_{0}=\xi_{0}$ is a constant.

Eq. (16) represents a bright soliton solution whereas Eq. (17) represents singular soliton solution and these are valid for

$\left(c^{2}-1\right)\left\{\beta-\left(c^{2}-1\right) \omega^{2}\right\}>0$.

Using Eqs. (8) and (11), we obtain the corresponding bright and singular soliton solutions of Eq. (2) as

$v(x, t)=\frac{2\left\{\beta\left(c^{2}+1\right)-\left(c^{4}-1\right) \omega^{2}\right\}}{c^{4}-1} \times \operatorname{sech}^{2}\left[\sqrt{\frac{\beta-\left(c^{2}-1\right) \omega^{2}}{c^{2}-1}}\left(x-c t-x_{0}\right)\right]$

and

$v(x, t)=-\frac{2\left\{\beta\left(c^{2}+1\right)-\left(c^{4}-1\right) \omega^{2}\right\}}{c^{4}-1} \times \operatorname{cosech}^{2}\left[\sqrt{\frac{\beta-\left(c^{2}-1\right) \omega^{2}}{c^{2}-1}}\left(x-c t-x_{0}\right)\right]$ 
For $\left(c^{2}-1\right)\left\{\beta-\left(c^{2}-1\right) \omega^{2}\right\}<0$, Eq. (1) will have periodic solutions given by

$u(x, t)= \pm \sqrt{\frac{2\left\{\beta\left(c^{2}+1\right)-\left(c^{4}-1\right) \omega^{2}\right\}}{\gamma\left(c^{2}-1\right)}} \times \sec \left[\sqrt{-\frac{\beta-\left(c^{2}-1\right) \omega^{2}}{c^{2}-1}}\left(x-c t-x_{0}\right)\right] \times e^{-i(k x-\omega t)}$

and

$u(x, t)= \pm \sqrt{\frac{2\left\{\beta\left(c^{2}+1\right)-\left(c^{4}-1\right) \omega^{2}\right\}}{\gamma\left(c^{2}-1\right)}} \times \operatorname{cosec}\left[\sqrt{-\frac{\beta-\left(c^{2}-1\right) \omega^{2}}{c^{2}-1}}\left(x-c t-x_{0}\right)\right] \times e^{-i(k x-\omega t)}$

Using Eqs. (8) and (11), the corresponding periodic solutions of Eq.(2) are given by

$v(x, t)=\frac{2\left\{\beta\left(c^{2}+1\right)-\left(c^{4}-1\right) \omega^{2}\right\}}{\left(c^{4}-1\right)} \times \sec ^{2}\left[\sqrt{-\frac{\beta-\left(c^{2}-1\right) \omega^{2}}{c^{2}-1}}\left(x-c t-x_{0}\right)\right]$

and

$v(x, t)=\frac{2\left\{\beta\left(c^{2}+1\right)-\left(c^{4}-1\right) \omega^{2}\right\}}{\left(c^{4}-1\right)} \times \operatorname{cosec}^{2}\left[\sqrt{-\frac{\beta-\left(c^{2}-1\right) \omega^{2}}{c^{2}-1}}\left(x-c t-x_{0}\right)\right]$

If we choose $a_{0}=-\frac{\left(c^{2}+1\right)\left\{\beta-\left(c^{2}-1\right) \omega^{2}\right\}^{2}}{2 \gamma\left(c^{2}-1\right)^{2}}$ in Eq. (15), then integrating with respect to U, we can obtain $U(\xi)$.

Substituting the expression for $U(\xi)$ thus obtained into Eq. (7), we obtain a dark and a singular soliton solutions of Eq.(1) as

$u(x, t)= \pm \sqrt{\frac{\beta\left(c^{2}+1\right)-\left(c^{4}-1\right) \omega^{2}}{\gamma\left(c^{2}-1\right)}} \times \tanh \left[\sqrt{-\frac{\beta-\left(c^{2}-1\right) \omega^{2}}{2\left(c^{2}-1\right)}}\left(x-c t-x_{0}\right)\right] \times e^{-i(k x-\omega t)}$

and

$u(x, t)= \pm \sqrt{\frac{\beta\left(c^{2}+1\right)-\left(c^{4}-1\right) \omega^{2}}{\gamma\left(c^{2}-1\right)}} \times \operatorname{coth}\left[\sqrt{-\frac{\beta-\left(c^{2}-1\right) \omega^{2}}{2\left(c^{2}-1\right)}}\left(x-c t-x_{0}\right)\right] \times e^{-i(k x-\omega t)}$

The dark and the singular soliton solutions in Eqs. (24) And (25) are valid for

$\left(c^{2}-1\right)\left\{\beta-\left(c^{2}-1\right) \omega^{2}\right\}<0$.

Using Eqs. (8) And (11), the corresponding dark and singular soliton solutions of Eq.(2) are given by

$v(x, t)=\frac{\beta\left(c^{2}+1\right)-\left(c^{4}-1\right) \omega^{2}}{\left(c^{4}-1\right)} \times \tanh ^{2}\left[\sqrt{-\frac{\beta-\left(c^{2}-1\right) \omega^{2}}{2\left(c^{2}-1\right)}}\left(x-c t-x_{0}\right)\right]$

and

$v(x, t)=\frac{\beta\left(c^{2}+1\right)-\left(c^{4}-1\right) \omega^{2}}{\left(c^{4}-1\right)} \times \operatorname{coth}^{2}\left[\sqrt{-\frac{\beta-\left(c^{2}-1\right) \omega^{2}}{2\left(c^{2}-1\right)}}\left(x-c t-x_{0}\right)\right]$.

For the condition

$\left(c^{2}-1\right)\left\{\beta-\left(c^{2}-1\right) \omega^{2}\right\}>0$, we will obtain periodic solutions.

\section{Conclusion}

In this study, the Trial Equation Method has been applied for finding some exact solutions of the Coupled Higgs Field Equation and thereby some soliton solutions and periodic solutions are obtained. It is seen that the performance of the method is quite effective and reliable and the application of the method can be extended to many other NLEEs arising in many areas of nonlinear sciences.

\section{References}

[1] LIU Cheng-Shi, Trial Equation Method to Nonlinear Evolution Equations with Rank Inhomogeneous: Mathematical Discussions and Its Applications, Commun. Theor. Phys. (Beijing, China), 2006, 45(2), 219-223. https://doi.org/10.1088/0253-6102/45/2/005.

[2] Triki, Houria; Wazwaz, A. M., Trial equation method for solving the generalized Fisher equation with variable coefficients, Phys. Lett. A, 2016. https://doi.org/10.1016/j.physleta.2016.02.002.

[3] Li, Yang, Application of Trial Equation Method for Solving the Benjamin Ono Equation, JAMP, 2014 , 2, 45 - 49. https://doi.org/10.4236/jamp.2014.23005.

[4] Biswas, A.; Yildrim, Y.; Yasar, E.; Triki, H.; Alshomrani, A. S.; Ullah, M. Z.; Zhou, Q.; Moshokoa, S. P.; Belic, M.; Optical soliton perturbation with full nonlinearity by trial equation method, Optik, 2018, 157, 1366 - 1375. https://doi.org/10.1016/j.ijleo.2017.12.113.

[5] Biswas, A.; Yildrim,Y.; Yasar, E.; Zhou,Q.; Alshomrani, A. S.; Moshokoa, S. P.; Belic, M.; Dispersive optical solitons with Schrödinger-Hirota model by trial equation method, Optik, 2018, 162, 35 - 41. https://doi.org/10.1016/j.ijleo.2018.02.058. 
[6] Alquran, M.; Katatbeh, Q.; Al-Shrida, B.; Applications of First Integral Method to Some Complex Nonlinear Evolution Systems, Appl. Math. Inf. Sci., 2015, 9(2), 825-831.

[7] Wazwaz, A. M.; Abundant soliton and periodic wave solutions for the coupled Higgs field equation, the Maccari system and the Hirota-Maccari system, Phys. Scr. 2012, 85, 065011 (10pp), stacks.iop.org/PhysScr/85/065011. https://doi.org/10.1088/0031-8949/85/06/065011.

[8] Abdelkawy, M.A.; Bhrawy, A.H.; Zerrad,E.; Biswas, A.; Application of Tanh Method to Complex Coupled Nonlinear Evolution Equations, Acta Phys. Pol. A, 2016, 129(3), 278 - 283. https://doi.org/10.12693/APhysPolA.129.278.

[9] Jabbari A.; Kheiri, H.; Bekir, A.; Exact solutions of the coupled Higgs equation and the Maccari system using He's semi-inverse method and $\left(\frac{G^{\prime}}{G}\right)$ expansion method, Comput. Math. Appl., 2011, 62, 2177 - 2186. https://doi.org/10.1016/j.camwa.2011.07.003.

[10] Khater, M. M. A.; Seadawy, A. R.; Lu, D.; Dispersive solitary wave solutions of new coupled Konno-Oono, Higgs field and Maccari equations and their applications, J. King Saud Univ. Sci., 2018, 30, 417 - 423. https://doi.org/10.1016/j.jksus.2017.11.003. 